\title{
A THREE-DIMENSIONAL ADAPTIVE WAVELET METHOD FOR FLUID-STRUCTURE INTERACTION
}

\author{
N.K.-R. Kevlahan ${ }^{1}$, O.V. Vasilyev ${ }^{2}$, D. Goldstein ${ }^{2}$, and A. Jay ${ }^{1,3}$ \\ kevlahan@mcmaster.ca \\ ${ }^{1}$ Department of Mathematics \& Statistics, McMaster University \\ Hamilton, ON, Canada \\ ${ }^{2}$ Mechanical $\&$ Aerospace Engineering, University of Colorado at Boulder, Boulder, CO, \\ USA \\ ${ }^{3}$ École MatMéca, Université de Bordeaux 1, Talence, France
}

\begin{abstract}
An adaptive collocation wavelet method for three-dimensional fluidstructure interaction at large Reynolds numbers is presented. This approach is shown to give accurate results with a reduced number of computational elements. The method is applied to two-dimensional flow past moving and fixed cylinders at $R e=10^{2}$ and $R e=10^{4}$, and to three-dimensional flow past a sphere at $R e=500$. This is the first three-dimensional calculation of flow past an obstacle using a dynamically adapted grid.
\end{abstract}

Keywords: Turbulence, fluid-structure interaction, wavelets, penalization

\section{Introduction}

One of the most practically important problems in aerodynamics is calculating moderate to high Reynolds number flow around solid obstacles of arbitrary shape. This problem arises in aerodynamics (e.g. turbulent flow over the wings and fuselage of airplanes), in off-shore drilling (e.g. water flow around riser tubes transporting oil from the sea bed to the surface), and in the wind engineering of buildings. In each case the primary difficulty arises from the need to calculate turbulent or transitional flow with boundary conditions on complicated domains. In addition, it may be important to allow for the obstacle to move or deform in response to the applied fluid forces (this motion in turn affects the flow).

In this paper we combine two mathematical approaches to calculate turbulent flow in complex domains. The first technique, the adap- 
tive wavelet method, tackles the problem of efficiently resolving a high Reynolds number flow in complicated geometries (where grid resolution should depend both on time and location). The second technique, Brinkman penalization, addresses the problem of efficiently implementing solid boundaries of arbitrary complexity.

Adaptive wavelet methods have been developed recently to solve the Navier-Stokes equations at moderate Reynolds numbers (e.g. Schneider et al., 1997; Vasilyev and Bowman, 2000; Vasilyev and Kevlahan, 2002; Griebel and Koster, 2002). The adaptive wavelet method is appropriate to turbulence since the wavelets (which are localized in both space and scale) adapt the numerical resolution naturally to the intermittent structure of turbulence at small scales. The wavelet method thus allows turbulent flows to be calculated with a greatly reduced number of modes and a well-controlled error. Furthermore, the computational cost is $O(\mathcal{N})$ (where $\mathcal{N}$ is the total number of wavelets actually used), which does not depend directly on the dimensionality of the problem. We employ a collocation wavelet method using second generation wavelets. This approach allows the order of the method to be varied easily (we generally use a 6th-order method). Another advantage of the collocation wavelet approach is that it is equally easy to implement in two or three dimensions. Indeed, the same code is used to do both two-dimensional and three-dimensional simulations presented here.

Parallel to the development of efficient wavelet codes for turbulence, we have been investigating the use of the Brinkman equation to simulate the presence of arbitrarily complex solid boundaries (Kevlahan and Ghidaglia, 2001). This technique allows boundary conditions to be enforced to a specified precision, without changing the numerical method (or grid) used to solve the equations. The main advantage of this method, compared to other penalization methods, is that the error can be estimated rigorously in terms of the penalization parameter. It can also be shown that the solution of the penalized equations converges to the exact solution in the limit as the penalization parameter tends to zero. Because this volume penalization is very simple and cheap to calculate, it is well-suited to moving obstacles. The adaptive wavelet method then allows the computational grid to following the moving obstacle, without the need for accelerating reference frames, or large areas of very fine grids.

The combination of the above two methods is applied here to the twoand three-dimensional Navier-Stokes equations. We have also developed a multilevel elliptic solver, based on the adapted multiscale wavelet grid, to solve the Poisson problem for the pressure at each timestep. Note that we do not use a subgrid-scale model: we resolve fully all significant 
length-scales. Thus, the wavelet grid follows the natural intermittency of the flow.

In $\S 1$ and $\S 2$ we briefly sketch the penalization and numerical methods. Some results for two- and three-dimensional flow past obstacles for Reynolds numbers up to $10^{4}$ are shown in $\S 3$, and in $\S 4$ we make some concluding comments. Note that this is the first time an dynamically adaptive wavelet method has been implemented for the threedimensional Navier-Stokes equations with obstacles.

\section{Brinkman penalization for complex geometries}

Incompressible fluid flow is described by the Navier-Stokes equations:

$$
\begin{aligned}
\frac{\partial \boldsymbol{u}}{\partial t}+(\boldsymbol{u}+\boldsymbol{U}) \cdot \nabla \boldsymbol{u}+\nabla P & =\nu \Delta \boldsymbol{u}, \\
\nabla \cdot \boldsymbol{u} & =0,
\end{aligned}
$$

where $\boldsymbol{U}$ is an imposed mean flow. We consider here the case where the fluid occupies the complement in $\Re^{3}$ of a set of $N$ obstacles $O_{i}$, $i=1, \ldots N$. The problem is solved on a rectangular computational domain $\Omega=\left[L_{11}, L_{21}\right] \times\left[L_{12}, L_{22}\right] \times\left[L_{13}, L_{23}\right]$ containing all obstacles. To these equations are added appropriate external (inflow, outflow and side) boundary conditions.

On the surface of the obstacles the velocity must satisfy the no-slip condition,

$$
\boldsymbol{u}+\boldsymbol{U}=\boldsymbol{U}_{o} \text { on } \partial O_{i}, \forall i
$$

where $\boldsymbol{U}_{o}$ is the velocity of the obstacle. Imposing these boundary conditions explicitly is difficult and computationally expensive when the obstacles have complicated shapes, move, or deform. To model the effect of the no-slip boundary conditions on the obstacles $O_{i}$ without explicitly imposing (3) we follow Angot et al., 1999 by replacing (1-3) by the following set of $L^{2}$-penalized equations

$$
\begin{aligned}
\frac{\partial \boldsymbol{u}_{\eta}}{\partial t}+\left(\boldsymbol{u}_{\eta}+\boldsymbol{U}\right) \cdot \nabla \boldsymbol{u}_{\eta}+\nabla P_{\eta}= & \nu \Delta \boldsymbol{u}_{\eta} \\
& -\frac{1}{\eta} \chi(\boldsymbol{x}, t)\left(\boldsymbol{u}_{\eta}+\boldsymbol{U}-\boldsymbol{U}_{o}\right), \\
\nabla \cdot \boldsymbol{u}_{\eta}= & 0,
\end{aligned}
$$

Note that equations (4-5) are valid in the entire domain $\Omega$ : the last term on the right hand side of (4) is a volume penalization of the flow inside the obstacle. Here $0<\eta \ll 1$ is a penalization coefficient and $\chi$ is the 
characteristic (or mask) function defining the obstacle geometry:

$$
\chi(\boldsymbol{x}, t)= \begin{cases}1, & \text { if } \boldsymbol{x} \in O_{i} ; \\ 0, & \text { otherwise }\end{cases}
$$

Angot, 1999 proved that the solution of the penalized equations (4-5) converges to that of the Navier-Stokes equations (1-2) with the correct boundary conditions (3) as $\eta \rightarrow 0$. For finite $\eta$ the error in the boundary conditions is $O\left(\eta^{1 / 2}\right)$.

In general, the obstacles may be fixed, or allowed to move (or even deform). We consider here the case where there is a single obstacle that is either fixed or moves like a harmonic oscillator forced by the fluid. We therefore couple the penalized Navier-Stokes equations (4-5) to a harmonic oscillator equation for the motion of the obstacle's centre of mass $\boldsymbol{x}_{o}$,

$$
m \frac{d^{2} \boldsymbol{x}_{o}}{d t^{2}}+b \frac{d \boldsymbol{x}_{o}}{d t}+k \boldsymbol{x}_{o}=\boldsymbol{F}(t),
$$

where $m$ is obstacle's mass, $b$ is the mechanical damping, $k$ is its natural frequency, and $\boldsymbol{F}(t)$ is the fluid force. Since $\boldsymbol{F}(t)$ is given by

$$
\boldsymbol{F}_{i}(t)=\boldsymbol{F}_{i}(\boldsymbol{u}(t))=\frac{1}{\eta} \int_{O_{i}}\left(\boldsymbol{u}+\boldsymbol{U}-\boldsymbol{U}_{o}\right) \mathrm{d} \boldsymbol{x} .
$$

the obstacle and fluid motions are fully and explicitly coupled.

\section{Numerical method}

We use an adaptive collocation wavelet method (Vasilyev and Bowman, 2000; Vasilyev and Kevlahan, 2002; Vasilyev, 2003) to dynamically adapt the grid to the solution, and to interpolate on the adapted grid. Derivatives are then calculated on the adapted grid using high-order finite differences (usually 6th-order). The grid is adapted at each time step by nonlinear wavelet filtering: only those points whose associated wavelet coefficients are greater than a threshold $\epsilon$ are retained. The solution may then be interpolated onto the adapted grid with an $L^{\infty}$ error of $O(\epsilon)$. Since the wavelet transform has $O(\mathcal{N})$ complexity (where $\mathcal{N}$ is the number of points in the adapted grid), the method is computationally efficient and scales well to large problems.

To allow for the change in the solution over one time step, nearest neighbours in position and scale are added to the adapted grid. Adding nearest neighbours in position corresponds to a CFL criterion of one. As the scales are dyadic, adding nearest neighbours in scale means allowing for the creation of scales twice as small via the quadratic nonlinearity of the Navier-Stokes equations. 
Because the wavelet basis is not divergence free, we employ the usual split-step method to make the velocity of the first half-step $\boldsymbol{u}^{*}$ divergence free. This Leray projection involves solving a Poisson equation for the pressure $P$,

$$
\nabla \cdot \nabla P=\frac{1}{\Delta t} \nabla \cdot \boldsymbol{u}_{*}
$$

Equation (9) is solved using a standard multilevel technique with Vcycles (Brandt, 1982). In our case, however, the grids on each level are provided by the adaptive wavelet multiresolution, which produces a natural adaptive method for the Poisson equation. Wavelets are also used to interpolate between levels. This is the first time a wavelet multilevel solver has been developed, and it is described fully in a companion publicationVasilyev and Kevlahan, 2003. Note that since it is based on the wavelet transform, the elliptic solver also has complexity $O(\mathcal{N})$.

Finally, we use a stiffly-stable 2nd-order time integration scheme that is semi-implicit for the advective term, and implicit for the penalization and diffusion terms.

\section{Results}

In this section we briefly present the results of some two- and threedimensional calculations. They have been selected to illustrate the effectiveness of the dynamically adapted grid, and the flexibility of the method. The results presented below used a tolerance of $\epsilon=10^{-4}$ for grid adaptivity, and the time step is chosen to maintain a CFL criterion of one.

The first example demonstrates the ability of the adaptive wavelet method to adapt the grid to fine-scale vortical structure. This result is interesting since although we actually solve the velocity form of the fluid equations, the grid points are distributed like the vortices of a (grid-free) vortex method. Figure 1 shows the vorticity and adapted grid for two-dimensional flow through a tightly packed periodic array of cylinders at $R e=10^{4}$. Note that very few points are required inside the obstacle, and the grid refines and coarsens as needed in order to resolve the vorticity. Only 66862 points out of a maximum of $896^{2}$ are used, which corresponds to a compression ratio of 12 .

In the second example we plot the drag, lift and amplitude of a twodimensional cylinder moving in response to fluid forces at $R e=10^{2}$. The oscillation amplitude $A=0.42$, lift amplitude $C_{L}=0.81$ and Strouhal frequency $S t=0.189$ are reasonably close to Shiels et al.'s(Shiels et al., 2001) vortex method values of $A=0.57, C_{L}=0.83, S t=0.194$.

The final example is flow through a periodic array of spheres at $R e=$ 500. This is a fully three-dimensional calculation, and shows the ability 

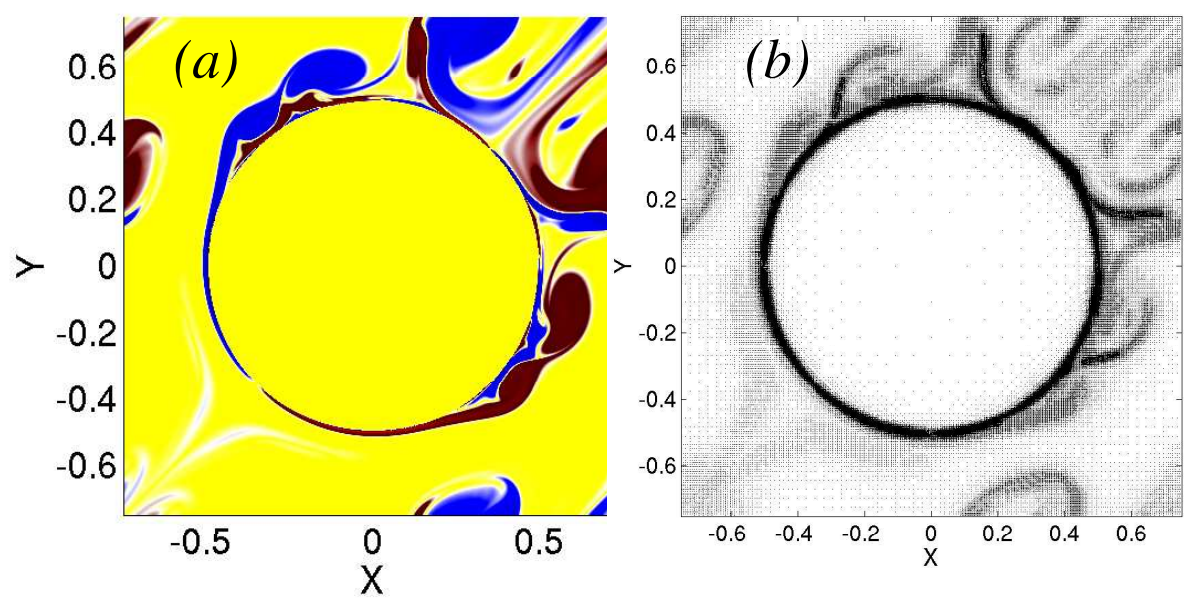

Figure 1. Two-dimensional periodic cylinder array at $R e=10^{4}, t=3.5$. (a) Vorticity. (b) Adapted grid.
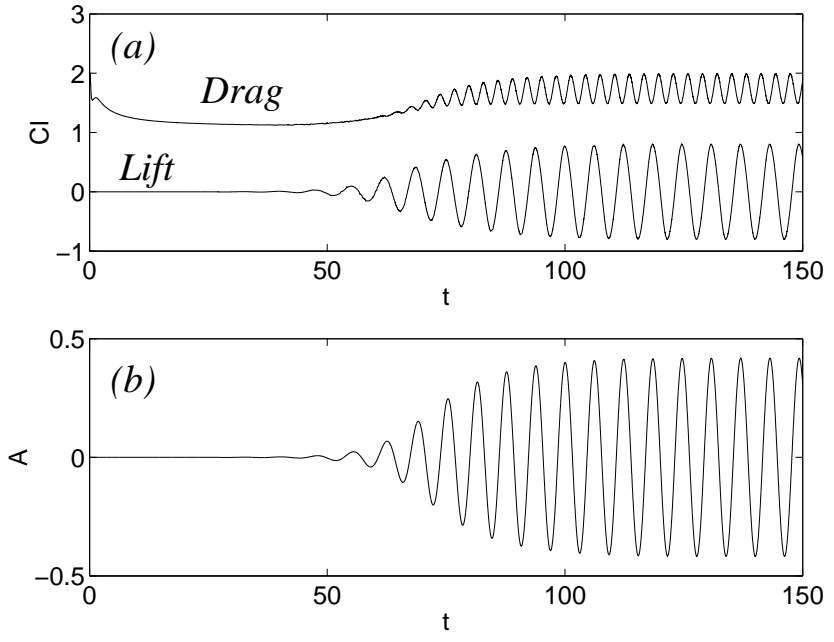

Figure 2. (a) Lift and drag for a moving cylinder at $R e=100$. Note that the average drag during the shedding phase is $C_{D}=1.74$, lift amplitude is $C_{L}=0.81$, and the Strouhal number is $S t=0.189$. (b) Cylinder displacement as a function of time (amplitude $A=0.42$ ). 

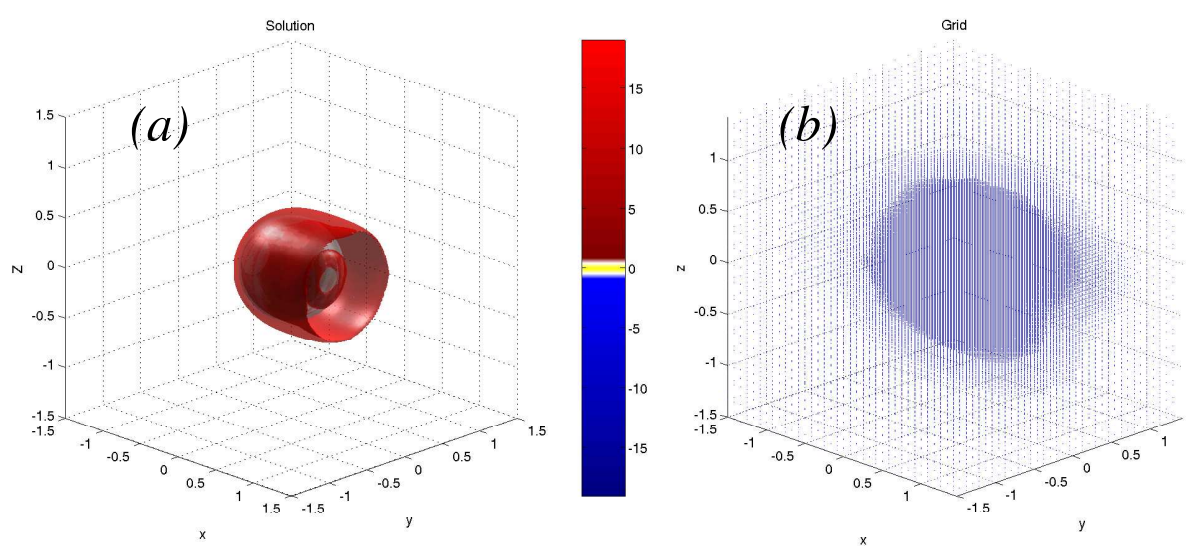

Figure 3. Flow past a sphere at $R e=500, t=3.4$ (looking upstream). (a) Isosurface of vorticity magnitude. (b) Computational grid.

of the method to efficiently solve the three-dimensional Navier-Stokes equations with obstacles. Figure 3 shows an isosurface of the vorticity magnitude, and the computational grid at $t=3.8$. Note that the flow is still at an early stage, so no instabilities have developed. The maximum resolution is $144^{3}$, but only 258000 , or $11.6 \%$, of the points are active. This example shows the importance of using a dynamically adaptive method in three-dimensional calculations.

\section{Conclusions}

In this paper we have presented a new method for calculating threedimensional flows at moderate to high Reynolds numbers with obstacles of arbitrary shape. The method uses an adaptive collocation wavelet method to dynamically adapted the grid to the flow, and as the basis of a multilevel solver for the associated Poisson equation for pressure.

We showed results for two-dimensional flow past a moving cylinder at $R e=10^{2}$ and a fixed cylinder at $R e=10^{4}$, and three-dimensional flow past a sphere at $R e=500$. This is the first three-dimensional calculation calculation of flow past an obstacle using a dynamically adapted grid.

Further three-dimensional test cases will be investigated in future work. In particular, we would like to determine if the number of active grid points is proportional to the Taylor scale $\left(\lambda=R e^{-1 / 2}\right)$, as is the case in two dimensions (Kevlahan and Vasilyev, 2003). If this is true, the overall computational complexity would scale like $R e$ which is much better than the classical estimate of $R e^{3}$ based on a uniform grid. 


\section{Acknowledgments}

The authors wish to thank P. Angot, P. Fabrie, J.-M. Ghidaglia, and C.H.K. Williamson for their help and advice. N. Kevlahan would like to thank NSERC for financial support, and SHARCNET for computational facilities.

\section{References}

Angot, P. (1999). Analysis of singular perturbations on the brinkman problem for fictitious domain models of viscous flows. Mathematical Methods in the Applied Science, 22:1395-1412.

Angot, P., Bruneau, C.-H., and Fabrie, P. (1999). A penalization method to take into account obstacles in viscous flows. Numerische Mathematik, 81:497-520.

Brandt, A. (1982). Guide to multigrid development. In Hackbusch, W. and Trottenberg, U., editors, Multigrid methods, volume 960 of Lecture Notes in Mathematics, pages 220-312. Springer-Verlag.

Griebel, M. and Koster, K. (2002). Multiscale methods for the simulation of turbulent flows. In Hirschel, E., editor, DFG/CNRS Workshop, Nice, 2001, Notes on Numerical Fluid Mechanics. Vieweg-Verlag.

Kevlahan, N. and Ghidaglia, J.-M. (2001). Computation of turbulent flow past an array of cylinders using a spectral method with brinkman penalization. Eur. J. Mech./B, 20:333-350.

Kevlahan, N. and Vasilyev, O. (2003). An adaptive wavelet collocation method for fluid-structure interaction at high reynolds numbers. SIAM J. Sci. Comput. Submitted.

Schneider, K., Kevlahan, N. K.-R., and Farge, M. (1997). Comparison of an adaptive wavelet method and nonlinearly filtered pseudo-spectral methods for twodimensional turbulence. Theoret. Comput. Fluid Dynamics, 9:191-206.

Shiels, D., Leonard, A., and Roshko, A. (2001). Flow-induced vibration of a circular cylinder at limiting structural parameters. J. Fluids Structures, 15:3-21.

Vasilyev, O. V. (2003). Solving multi-dimensional evolution problems with localized structures using second generation wavelets. Int. J. Comp. Fluid Dyn., Special issue on high-resolution methods in Computational Fluid Dynamics, 17(2):151168.

Vasilyev, O. V. and Bowman, C. (2000). Second generation wavelet collocation method for the solution of partial differential equations. J. Comput. Phys., 165:660-693.

Vasilyev, O. V. and Kevlahan, N. K.-R. (2002). Hybrid wavelet collocation-brinkman penalization method for complex geometry flows. Int. J. Num. Meth. Fluids, 30:531538.

Vasilyev, O. V. and Kevlahan, N. K.-R. (2003). An adaptive multilevel wavelet collocation method for elliptic problems. In preparation. 